\title{
Raman study of self-assembled InAs/InP quantum wire stacks with varying spacer thickness
}

\author{
T. Angelova, ${ }^{1}$ A. Cros, ${ }^{1, a)}$ A. Cantarero, ${ }^{1}$ D. Fuster, ${ }^{2}$ Y. González, ${ }^{2}$ and L. González ${ }^{2}$ \\ ${ }^{1}$ Institut de Ciència de Materials, Universitat de València, P.O. Box 22085, E-46071 València, Spain \\ ${ }_{2}^{2}$ Institut de Microelectrónica de Madrid (CNM-CSIC), Isaac Newton 8, Tres Cantos, 28760 Madrid, Spain
}

(Received 9 April 2008; accepted 31 May 2008; published online 6 August 2008)

\begin{abstract}
Self-assembled InAs/InP (001) quantum wire stacks have been investigated by means of Raman scattering. The characteristics of the observed vibrational modes show clear evidence of confinement and atomic intermixing between As and $\mathrm{P}$ atoms from the wire and the spacer. The change in the intermixing with spacer layer thickness and growth temperature is investigated. Likewise, the effect of annealing on the exchange of As and P atoms is also studied. Resonance effects in confined and interface phonons are discussed for excitation in the vicinity of the InAs $E_{1}$ critical point. Finally, the energy of the interface modes is related to the structural characteristics of the wires by comparing the experimental data with a lattice dynamic calculation based on the dielectric continuum model. (C) 2008 American Institute of Physics. [DOI: 10.1063/1.2963703]
\end{abstract}

\section{INTRODUCTION}

The interest in the optical properties of self-assembled InAs/InP quantum wires (QWrs) and quantum dots (QDs) grown in the Stranski-Krastanov mode has been motivated by their promising applications in optoelectronic devices operating in the $1.30-1.55 \mu \mathrm{m}$ wavelength range employed in telecommunication systems. ${ }^{1,2}$ More specifically, it has been demonstrated that in InAs/InP QWrs and QDs, the combination of strain, carrier confinement, and control of As/P substitution can be used to tune the band gap and photon emission in the near-infrared spectral region. ${ }^{3}$

Besides photoluminescence studies, several investigations on Raman scattering (RS) in InAs/InP nanostructures (mainly QDs) have been performed in the past. ${ }^{4,5}$ These have led to the identification of As/P intermixing through the vibrational structure observed in the dots, as well as the determination of the compressive strain in the nanostructures via the blueshift of their phonon wavenumbers when compared to those of bulk InAs. ${ }^{6,7}$ Little work has been devoted to the study of the vibrational properties of self-assembled QWrs, although their distinctive two-dimensional confinement presents peculiarities when compared to the Raman spectra of QDs. ${ }^{8}$

In the present work we make use of RS for the investigation of the vibrational spectra of stacks of InAs/InP QWrs with various spacer thicknesses. The work is organized as follows. In Sec. II we describe the conditions for sample growth and the experimental setup. In Sec. III we discuss the results of our measurements and modeling. Conclusions are drawn in Sec. IV.

\section{EXPERIMENTAL DETAILS}

Two series of InAs QWrs grown on (001) InP by solidsource molecular beam epitaxy have been investigated in this work. The first series was grown at a temperature of $380{ }^{\circ} \mathrm{C}$

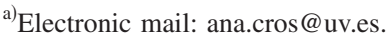

[denoted in what follows as low temperature (LT)] and consists of three samples denoted as LT1-LT3. The second series, comprising four samples, was grown at a higher temperature $\left[515^{\circ} \mathrm{C}\right.$, denoted in what follows as high temperature (HT), samples HT0-HT3]. The characteristics of the samples are detailed in Table I and differ mainly in the thickness of the InP spacer. High-resolution transmission electron microscopy shows that the QWrs are oriented along the $[1 \overline{1} 0]$ direction. ${ }^{4}$ Concerning their size, the wires of the HT series were found to be nearly a factor of 3 higher than those of the LT series, with average sizes of (width/height) $12.8 \mathrm{~nm} / 1.2 \mathrm{~nm}$ for the HT series and $10.3 \mathrm{~nm} / 3.2 \mathrm{~nm}$ for the LT series. In both series the mean length of the wires is $1 \mu \mathrm{m} .{ }^{9}$ The structural characteristics of the samples have been summarized in Table I.

RS measurements were carried out in a Jobin-Yvon T64000 triple spectrometer equipped with a confocal microscope and a nitrogen-cooled charge-coupled device detector. The spectra were taken in backscattering geometry in the $z(x, x) \bar{z}, z(y, y) \bar{z}$, and $z(x, y) \bar{z}$ polarization configurations $(x$

TABLE I. Structure and growth parameters of the self-assembled InAs/InP QWr stacks. The number of stacked periods, the thickness of the InP spacer, and the growth temperature are indicated for each sample.

\begin{tabular}{c|c|c|c|c}
\hline \hline \multirow{2}{*}{ Series } & Sample & Periods & $\begin{array}{c}d(\mathrm{InP}) \\
(\mathrm{nm})\end{array}$ & $\begin{array}{c}T_{\text {growth }} \\
\left({ }^{\circ} \mathrm{C}\right)\end{array}$ \\
\hline \multirow{4}{*}{ LT } & LT1 & 7 & 5 & 380 \\
\cline { 2 - 5 } & LT2 & 7 & 10 & 380 \\
\cline { 2 - 5 } & LT3 & 6 & 20 & 380 \\
\hline \multirow{3}{*}{ HT } & HT0 & 7 & 3 & 515 \\
\cline { 2 - 5 } & HT1 & 7 & 5 & 515 \\
\cline { 2 - 5 } & HT2 & 7 & 10 & 515 \\
\cline { 2 - 5 } & HT3 & 7 & 20 & 515 \\
\hline \hline
\end{tabular}




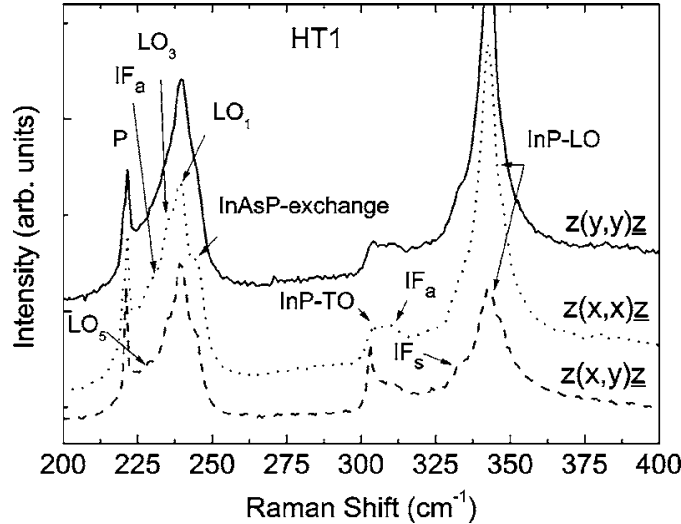

FIG. 1. Micro-Raman spectra of InAs/InP QWrs from sample HT2 in parallel and crossed polarizations along the directions [110] $(x)$ and $[1 \overline{1} 0](y)$.

$=[110], y=[1 \overline{1} 0]$, and $z=[001])$. A $100 \times$ microscope objective was used to focus the light to a $1 \mu \mathrm{m}$ spot on the sample surface and collect the scattered signal to the spectrometer. The samples were excited by the $488 \mathrm{~nm}$ line of an $\mathrm{Ar}^{+}$laser and the power density was kept at $200 \mathrm{~W} \mathrm{~cm}^{-2}$ to avoid undesired sample heating. In the measurements under resonant conditions, several lines of an argon ion laser comprising a wide energy range $(2.40,2.47,2.50,2.54,2.60,2.62$, 2.71 , and $2.73 \mathrm{eV}$ ) were used. The annealing of the samples at different temperatures was done in a Linkam TS 1500 heating stage. The Raman spectra were first recorded at room temperature. Next, the hot stage was raised from RT $\left(25^{\circ} \mathrm{C}\right)$ to $225{ }^{\circ} \mathrm{C}$ at a rate of $20^{\circ} \mathrm{C} / \mathrm{min}$. After $10 \mathrm{~min}$ at this temperature, the sample was cooled down to RT, and a spectrum was recorded. This procedure was repeated by heating up to $325,425,525,625,725,825$, and $875{ }^{\circ} \mathrm{C}$, recording the corresponding Raman spectra at RT for each annealing temperature.

\section{EXPERIMENTAL RESULTS AND DISCUSSION}

\section{A. Analysis of the Raman modes and influence of the spacer thickness}

Let us examine first the micro-Raman spectra of sample HT1 recorded using $2.54 \mathrm{eV}(488 \mathrm{~nm})$ as excitation energy, shown in Fig. 1 for the various polarization configurations. The plasma line at $221.0 \mathrm{~cm}^{-1}$ marked as $P$ in the figure has been used to calibrate the spectra with an uncertainty below $\pm 0.4 \mathrm{~cm}^{-1}$. Taking into account the selection rules of RS in bulk zinc-blende semiconductors in backscattering geometry along a (001) surface, longitudinal optical (LO) phonons should be only allowed in parallel polarization, while transverse optical (TO) modes should be forbidden. We found that the normal vibrations of both wires and spacer materials violate these selection rules. Concerning interface (IF) vibrations, the current theory ${ }^{10}$ predicts four modes. A pair of symmetric and antisymmetric modes can be assigned to each material at the IF. Symmetric IF modes $\left(\mathrm{IF}_{s}\right)$ are allowed in crossed polarization and antisymmetric ones $\left(\mathrm{IF}_{a}\right)$ in parallel polarization, but their observation requires a nonzero wavevector component parallel to the IF. As can be seen in Fig. $1, \mathrm{IF}_{a}$ and $\mathrm{IF}_{s}$ modes are observed in both polarization configurations, although they are weaker in the forbidden one. Various effects may be the cause of the violation of the selection rules. First, the scattering of the light may take place along different crystallographic orientations due to its interaction with various crystalline facets. This effect has been observed in similar heterostructures. ${ }^{7,8}$ Second, Raman measurements were taken near resonance to the $E_{1}$ band gap of the wires, and it is well known that the Fröhlich interaction near resonance may cause the breakdown of the selection rules for polar phonon modes.

The observed peaks may be grouped in a low energy $\left(\omega<250 \mathrm{~cm}^{-1}\right.$, InAs-like $)$ and a high energy $(\omega$ $>300 \mathrm{~cm}^{-1}$, InP-like) region. Let us consider first the characteristics of the high energy side. The peaks at 342 and $303 \mathrm{~cm}^{-1}$ can be attributed to the LO and TO phonon modes of the InP barrier, respectively. Their Raman shifts are very similar to those found in previous reports for the bulk material. ${ }^{11}$ As commented before, the broad structures observed around $310 \mathrm{~cm}^{-1}$ and the peak at $333 \mathrm{~cm}^{-1}$ can be attributed to antisymmetric and symmetric IF modes, respectively, in which the $\mathrm{P}$ atoms have the maximum amplitude of vibration. This assignment will be discussed later based on a comparison with a theoretical calculation. ${ }^{12}$ The low energy region of the spectra, corresponding to the InAs wires, presents a richer structure. Three phonon modes between 200 and $240 \mathrm{~cm}^{-1}$ appear in both polarization configurations and are assigned as $\mathrm{LO}_{1}, \mathrm{LO}_{3}$, and $\mathrm{LO}_{5}$ modes. According to their frequency and taking into account the bulk InAs dispersion curve, ${ }^{13}$ they can be classified as LO confined modes. ${ }^{14}$ This assignment is further confirmed by the resonant behavior of these modes when excited with energy close to the $E_{1}$ transition of InAs (Sec. III C). The energy of the $\mathrm{LO}_{1}$ mode corresponding to the InAs wire is the same in both series and is nearly coincident with the LO phonon mode of bulk InAs $\left(239.8 \mathrm{~cm}^{-1}\right)$, indicating that the QWrs are almost relaxed. The broad structure at $234 \mathrm{~cm}^{-1}$ labeled as $\mathrm{IF}_{a}$ corresponds to an antisymmetric IF mode in which the As atoms have a large oscillation amplitude in comparison to $\mathrm{P}$ atoms. This assignment will be discussed later in combination with the calculations and is supported as well by its behavior under resonant excitation. The peak denoted as InAsP exchange is observed in all polarization configurations but appears more clearly in $z(y, y) \underline{z}$, that is, when the polarization of the incident and the scattered light lies along the QWr direction. It has been attributed to the exchange of As and $\mathrm{P}$ atoms during the formation of the QWrs (Ref. 7) and will be discussed below in relation to the effect of annealing, spacer thickness, and growth temperature.

Figure 2(a) shows the comparison of the micro-Raman spectra of samples HT3 and LT3 recorded in the $z(x, x) \underline{z}$ polarization. Both series display the same peaks, but their intensities and frequencies differ. Concerning the peaks related to InP (high energy side), the main difference corresponds to the intensity of the $\mathrm{IF}_{a}$ mode, which is larger in the LT series than that in the HT series. The same difference can also be observed in the $\mathrm{IF}_{a}$ mode related to InAs at $234 \mathrm{~cm}^{-1}$, much more pronounced in the LT series. These differences reflect the larger amount of material occupying interfacial positions in the QWrs of the LT series due to their 

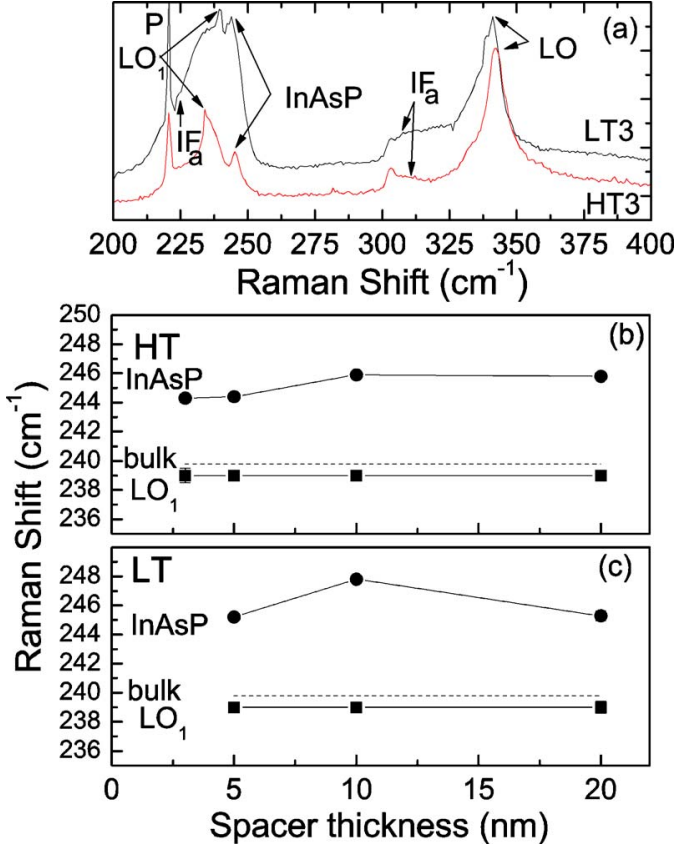

FIG. 2. (Color online) (a) Raman spectra samples LT3 and HT3. [(b) and (c)] Spacer thickness dependence of the frequency of the $\mathrm{LO}_{1}$ and IF vibrational modes of InAs/InP QWrs of both the LT and HT series. The frequency of the LO phonon mode of bulk InAs is indicated for comparison.

larger size. Concerning the dependence of the modes on spacer thickness, it is found that the intensity of the InP LO mode increases with the thickness of the spacer since the detected signal is proportional to the scattering volume. This demonstrates that the signal arises mostly from the spacer and not from the InP substrate. On the other hand the intensity of the $\mathrm{LO}_{1}$ mode of the QWrs does not change with spacer thickness, consistent with the constant amount of InAs in the samples. Concerning the frequency of the modes, its variation with spacer thickness is presented in Figs. 2(b) and 2(c) for samples of the HT and LT series, respectively. The changes are only significant for the InAsP-exchange mode. For the HT series, the frequency of the mode shifts upward $2 \mathrm{~cm}^{-1}$ for spacer thicknesses larger than $5 \mathrm{~nm}$. In the LT series this shift is only observed for the LT2 sample (10 $\mathrm{nm}$ spacer thickness). The blueshift is an indication of a larger $\mathrm{P}$ content in wires separated by a larger amount of InP. Considering the energy of the $\operatorname{InAs}_{x} P_{1-x}$ mode, its composition changes from $x>0.6$ for small spacer thicknesses to $x$ $<0.6$ for a spacer thickness of $20 \mathrm{~nm}$ (HT series). Due to the slow variation in the frequency of the InAsP phonon mode with $\mathrm{P}$ content, it is difficult to evaluate $x$ more precisely. In general, in our results, the intermixing is also observed to be larger in the samples grown at higher temperatures, in accordance with the work of Fuster et al. ${ }^{4}$ However, sample LT2 displays larger P content than expected. The influence of the size of the QWrs on its optical properties is observed in the Raman spectra when the polarization of the incident light is parallel (along $y=[1 \overline{1} 0]$ ) and perpendicular (along $x=[110]$ ) to the QWr direction. The peaks associated with LO and InAsP phonons experience a small redshift when the polarization is changed, the frequency change being larger for the InAsP mode $\left(1-2 \mathrm{~cm}^{-1}\right)$. As shown in Figs. 3(a) and 3(b)

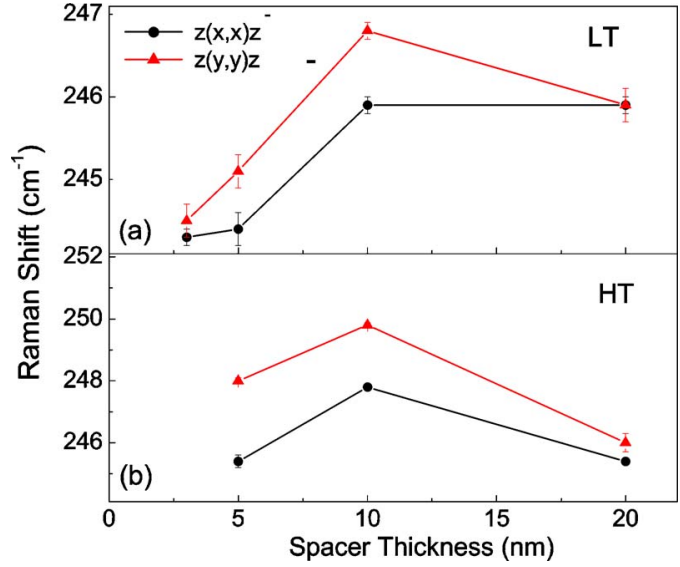

FIG. 3. (Color online) Raman shift in the InAsP phonon mode as a function of the spacer thickness for (a) the LT and (b) the HT series. The incident and scattered polarizations are either perpendicular (dots) or parallel (triangles) to the QWr direction.

for LT and HT series, respectively, this anisotropy, characteristic of the nanostructure and absent in bulk and QDs, is highest for samples with spacer thicknesses $d(\operatorname{InP})=5 \mathrm{~nm}$ and $d(\mathrm{InP})=10 \mathrm{~nm}$. It is not clear if this effect can be ascribed to the coupling of the LO and TO phonon modes in the QWrs, as predicted by Comas et al. ${ }^{15}$ In any case, the observed anisotropy is in accordance with the work reported by Gippius et al., ${ }^{16}$ where a strong linear polarization of the Raman signal along the wire axis in uncapped QWrs is observed. In that case, the anisotropy was ascribed to the spatial distribution of the electric component of the electromagnetic field in the vicinity of the QWr. ${ }^{16}$

\section{B. Effect of annealing on the As/P exchange}

Usually the InAs/InP quantum structures are fabricated at low substrate temperatures of $\sim 500{ }^{\circ} \mathrm{C}$ or lower. However, the temperature must be increased to grow high-quality InAs caps and InAsP layers, which are essential for lightemitting devices. Therefore, the effects of HT annealing on quantum structures are important and many works indicated that HT annealing introduces severe changes in the structure shape, size, and composition of the wires. ${ }^{17}$ Since annealing is known to favor As/P exchange at the nanowire surface, we have analyzed the changes induced by annealing in the vibrational modes of the QWrs. Figure 4(a) shows the Raman spectra of sample HT1, as grown and after annealing at 525 and $875{ }^{\circ} \mathrm{C}$ (see details of the annealing procedure in Sec. II) recorded in the $z(x, x) \underline{z}$ configuration. The spectra were normalized to the InP LO phonon mode for comparison. An important increase in the intensity of the mode related to InAsP is observed for annealing temperatures higher than $875^{\circ} \mathrm{C}$, with a simultaneous redshift in the energy of the LO, IF, and InAsP phonon modes [Figs. 4(b)-4(d)]. These variations can be explained by considering an increase in the $\mathrm{P}$ diffusion length inside the wires, with a small decrease in their mean $\mathrm{P}$ content. At the same time, the size of the remaining InAs material decreases, with a consequent redshift in the InAs LO and IF modes due to confinement effects. 

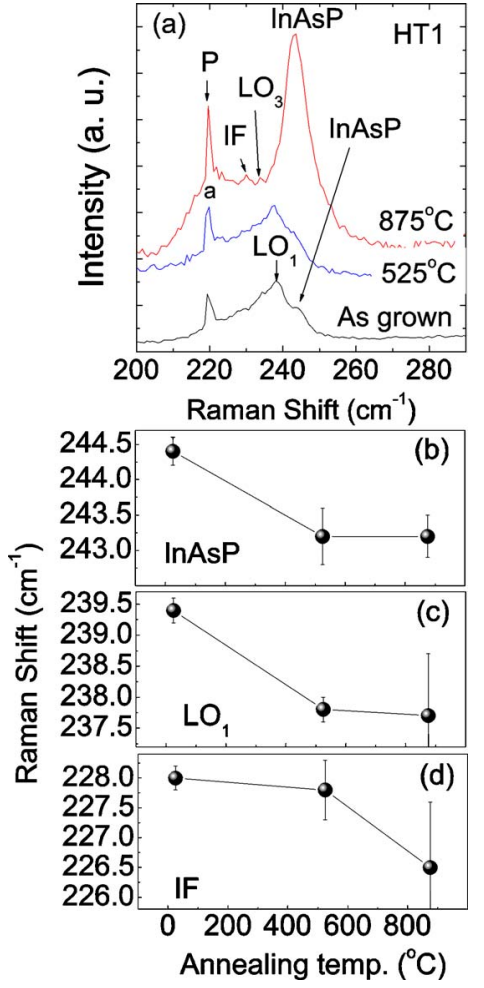

FIG. 4. (Color online) (a) Raman spectra of the as-grown and annealed InAs/InP QWrs from sample HT1 under parallel polarization. [(b)-(d)] Raman peak frequency as a function of annealing temperature for InAs $\mathrm{LO}_{1}$, IF phonon modes, and InAsP phonons of the alloy.

From the Raman shift in the InAsP-exchange mode, we estimate $^{18,19}$ that the concentration of As in the InAsP alloy after HT annealing is around $65 \%$.

\section{Resonant Raman in the vicinity of the InAs $E_{1}$ critical point}

Finally, we have analyzed the evolution of the Raman peaks under various excitation energies around the $E_{1}$ critical point of InAs located at $2.61 \mathrm{eV} .^{13}$ Figure 5(a) shows the spectra corresponding to sample LT2 taken in parallel polarization. A resonance enhancement of the $\mathrm{LO}_{1}$ phonon mode takes place in the vicinity of $2.5-2.62 \mathrm{eV}$, as can be observed from the evolution of the intensity of the mode with excitation energy shown in Fig. 5(b). The intensity of the InAs IF mode increases as well, but it does not present a clear maximum in this energy interval, indicating that the resonance may occur at somewhat higher energies for this mode. This can be understood by taking into account the mixed InAs/InP nature of the vibration and considering that the $E_{1}$ electronic transition of bulk InP is higher in energy $[3.1 \mathrm{eV}$ at $300 \mathrm{~K}$ (Ref. 5)].

\section{Model calculation for the interface phonons in an elliptical quantum wire: Application to the InAs/ InP (001) system}

The interpretation of the Raman spectra presented above and the assignment of the observed phonons may be improved by the use of a theoretical model ${ }^{12}$ that takes into account the size and shape of the QWrs and allows the cal-
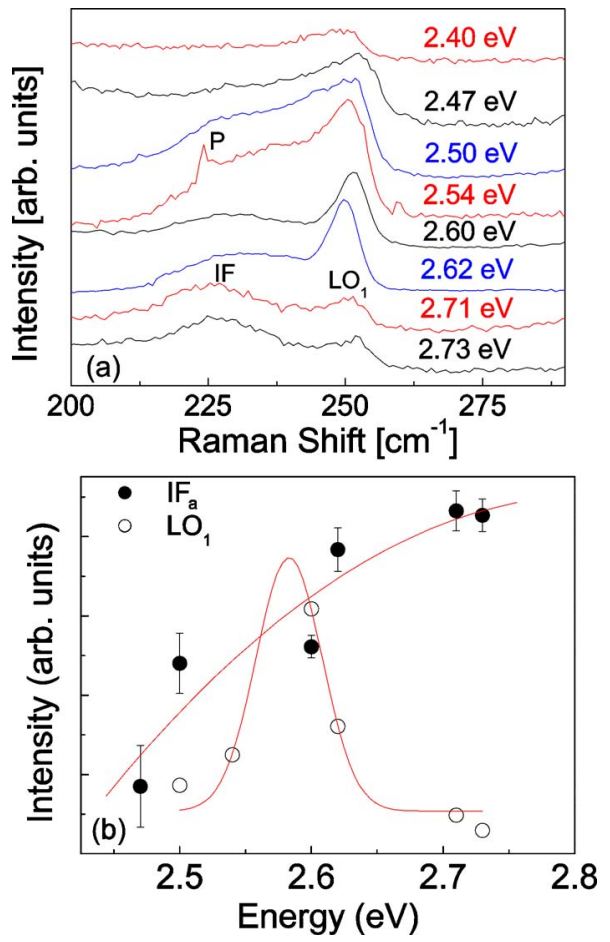

FIG. 5. (Color online) (a) Micro-Raman spectra of InAs/InP QWrs recorded at room temperature with several laser wavelengths for sample LT2. (b) Intensity dependence of the $\mathrm{LO}_{1}$ and IF phonon modes.

culation of the energy of the IF phonon modes. Due to their small dimensions, phonon modes of semiconductor QWrs are strongly affected by confinement, giving rise to confined and IF modes. ${ }^{14,15}$ To relate the energy of the IF modes to the structural characteristics of the wires, we have made use of the anisotropic dielectric continuum model. ${ }^{12}$ This model will be applied to a system consisting of a single wire with dielectric function $\varepsilon_{1}$ embedded in a second material with dielectric function $\varepsilon_{2}$. The dielectric function of the system can be written as

$$
\varepsilon_{i}(\omega)=\varepsilon_{i}(\infty) \frac{\omega^{2}-\omega_{\mathrm{LO}, i}^{2}}{\omega^{2}-\omega_{\mathrm{TO}, i}^{2}}, \quad i=1,2,
$$

where $i=1$ corresponds to the material of the QWrs and $i$ $=2$ to that of the barrier. For an infinite wire of elliptical cross section, with major axis $R$ and minor axis $r$, the potential can be separated in elliptical coordinates $(u, v)$ in an angular $[V(v)]$ and a radial $[U(u)]$ contribution,

$$
\begin{aligned}
& \frac{d^{2} V}{d v^{2}}+(\alpha-2 q \cos 2 v) V=0, \\
& \frac{d^{2} U}{d u}-(\alpha-2 q \cosh 2 u) U=0,
\end{aligned}
$$

where the parameter $\alpha$ is quantized and is related to the phonon eigenvalues for a given phonon wave vector $q^{2}$ $=k^{2}\left(r^{2}-R^{2}\right) / 4<0$. The solutions to these equations fall into four different symmetry classes depending on the sign of the potential upon reflection at the IF. According to this symmetry, the $m$ th eigenvalue of $\alpha\left(\alpha_{m}\right)$ will correspond to a symmetric or antisymmetric IF mode, and its corresponding 
TABLE II. Parameters used to calculate the dispersion curves of Fig. 5.

\begin{tabular}{l|c|c|c}
\hline \hline Material (Ref. 22) & $\varepsilon_{\infty}$ & $\begin{array}{c}\omega_{\mathrm{TO}} \\
\left(\mathrm{cm}^{-1}\right)\end{array}$ & $\begin{array}{c}\omega_{\mathrm{LO}} \\
\left(\mathrm{cm}^{-1}\right)\end{array}$ \\
\hline InAs & 12.3 & 218.8 & 239.8 \\
\hline $\mathrm{InP}$ & 9.61 & 304 & 345 \\
\hline \hline
\end{tabular}

eigenfunction is given by a periodic Mathieu function, ${ }^{20,21}$ finite near $u=0$. The phonon dispersion relations at $k=0$ are given by

$$
\frac{\varepsilon_{1}\left(\omega_{m}\right)}{\varepsilon_{2}\left(\omega_{m}\right)}=\frac{(R-r)^{m} \pm(R+r)^{m}}{(R-r)^{m} \mp(R+r)^{m}},
$$

where $m$ is a positive integer for symmetric and antisymmetric IF phonon modes. The last equation indicates that the frequency of the IF phonons will depend only on the $r / R$ ratio. For a wire with an elliptical cross section, the frequencies $\omega_{m}(k)$ of the IF modes lie in the frequency region of each material compound constituting the system (wire and spacer), with $\omega_{\mathrm{TO}, 1}<\omega_{m, 1}<\omega_{\mathrm{LO}, 1}$ and $\omega_{\mathrm{TO}, 2}<\omega_{m, 2}<\omega_{\mathrm{LO}, 2}$.

For comparison with the samples studied in this work, Eq. (4) has been solved for the case of an InAs elliptical wire, taking $R$ and $r$ of the LT and HT series as given by the atomic force microscopy characterization of the samples and with InP as the barrier material. The parameters given in Table II have been used for the calculations.

In order to identify the optical vibrations responsible for the three structures near 230,310, and $333 \mathrm{~cm}^{-1}$ observed (see Fig. 1), we have calculated the IF phonon mode energy as a function of aspect ratio $(r / R)$ for relaxed QWrs. The full and dashed lines in Fig. 6 show the dispersion of the IF phonon modes for an isolated InAs/InP QWr in comparison with the experimental results (dots). As expected, one pair of symmetric and antisymmetric phonon modes lies between the LO and TO frequencies of the bulk material that forms the wires, and the other pair lies between the LO and TO modes of the barrier. Frequencies of the IF phonon modes with $m>0$ are very close to those with $m=0$ and have not been included in Fig. 6.

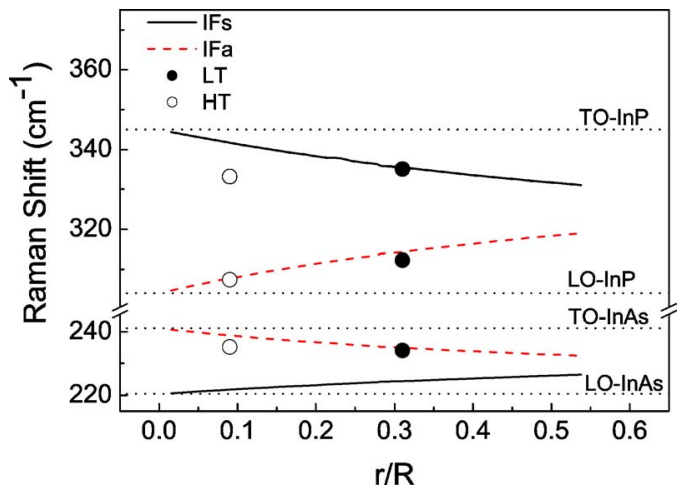

FIG. 6. (Color online) Frequencies of the IF optical phonons with $m=0$ for InAs QWrs with elliptical cross section embedded in an InP substrate as a function of the wire aspect ratio $r / R$. The points present the experimental values of the IF phonon modes of the HT and LT series of the samples with $d=5 \mathrm{~nm}$.
Within each series, the frequency of the IF modes does not change appreciably, in agreement with the fact that the aspect ratio of the QWrs remains almost constant when the thickness of the spacer is changed. Therefore, in Fig. 6 we have only included the values corresponding to samples LT1 and HT1. Both antisymmetric and symmetric IF phonons were observed for the InP barrier in the experiment. For the InAs QWrs, the symmetric mode is hidden behind a laser plasma line and it had not been possible to discriminate it. However, a small shoulder in the low energy side of the plasma line indicates its presence around $222 \mathrm{~cm}^{-1}$ [see Fig. $1, z(x, x) z$ configuration]. For the LT series the calculated IF vibrations are in excellent agreement with the observed Raman peaks. Concerning the HT series, the theoretical result overestimates the value of the InAs related IF phonon (low energy branch). This small discrepancy may be understood by considering the different sources of phonon frequency shift in the wire material. For a perfect heterostructure two contributions should be considered: An in-plane compressive strain of the wire results in an upward frequency shift. On the other hand, confinement effects downshift the phonon frequency due to the negative dispersion of the LO phonons. $^{23,24}$ The experimental results show that the QWrs are almost relaxed, so that we can rule out the first effect. The downshift experienced by the phonon mode of the HT series can be thus attributed to the larger phonon confinement effect for this series. In the LT series, due to its larger height, this effect is very small.

\section{CONCLUSIONS}

In this work, we have investigated the vibrational modes of InAs/InP QWrs by RS. The QWrs LO and TO modes were found to violate the expected bulk selection rules while the IF phonons follow them. The QWr IF mode frequencies are in good agreement with the results of the dielectric continuum model, with a more significant influence of confinement in the HT series than in the LT one. The P/As atomic exchange reflects the presence of a LO mode related to the ternary alloy InAsP. The P content of this alloy is larger for samples of the HT series. After annealing, the amount of alloy present in the samples increases, while its $\mathrm{P}$ mean content decreases slightly. At the same time, there is a decrease in the size of the wires. The resonance observed for the InAs $\mathrm{LO}_{1}$ and InAs IF modes in the vicinity of the $E_{1}$ critical point reinforces their assignment.

\section{ACKNOWLEDGMENTS}

The authors are thankful to the Ministry of Education and Science of Spain (Project No. MAT2006-01825, FEDER), the Generalitat Valenciana, and the European Network SANDIE (Grant No. NMP4-CT-2004-500101) for financial support.

${ }^{1}$ L. González, J. M. García, R. García, F. Briones, J. Martínez-Pastor, and C. Ballesteros, Appl. Phys. Lett. 76, 1104 (2000).

${ }^{2}$ R. Schwertberger, D. Gold, J. P. Reithmaier, and A. Forchel, J. Cryst. Growth 251, 248 (2003).

${ }^{3}$ P. J. Poole, R. L. Williams, L. Lefebvre, and S. Moisa, J. Cryst. Growth 257, 89 (2003). 
${ }^{4}$ D. Fuster, M. González, L. González, Y. González, T. Ben, A. Ponce, S. I. Molina, and J. Martínez-Pastor, Appl. Phys. Lett. 85, 1424 (2004).

${ }^{5}$ J. F. Girard, C. Dion, P. Desjardins, C. N. Allen, P. J. Poole, and S. Raymond, Appl. Phys. Lett. 84, 3382 (2004).

${ }^{6}$ L. Chu, A. Zrenner, M. Bichler, G. Böhm, and G. Abstreiter, Appl. Phys. Lett. 77, 3944 (2000).

${ }^{7}$ C. K. Chia, S. J. Chua, S. Tripathy, and J. R. Dong, Appl. Phys. Lett. 86, 051905 (2005).

${ }^{8}$ W. Lei, Y. H. Chen, B. Xu, X. L. Ye, Y. P. Zeng, and Z. G. Wang, Nanotechnology 16, 1974 (2005).

${ }^{9}$ D. Fuster, Ph.D. thesis, University of Valencia, Spain, 2006.

${ }^{10}$ M. Cardona and G. Günterodt, Light Scattering in Solids V (Springer, Berlin, 1989).

${ }^{11}$ A. Mooradian and G. B. Wright, Solid State Commun. 4, 431 (1966).

${ }^{12}$ P. A. Knipp and T. L. Reinecke, Phys. Rev. B 45, 9091 (1992).

${ }^{13}$ R. Carles, N. Saint-Cricq, J. B. Renucci, A. Zwick, and M. A. Renucci, Phys. Rev. B 22, 6120 (1980).

${ }^{14}$ J. Groenen, C. Priester, and R. Carles, Phys. Rev. B 60, 16013 (1999).

${ }^{15}$ F. Comas, C. Trallero-Giner, and A. Cantarero, Phys. Rev. B 47, 7602
(1993).

${ }^{16}$ N. A. Gippius, S. G. Tikhodeev, J. Rubio, J. M. Calleja, P. Ils, A. Forchel, and V. D. Kulakovskii, Phys. Status Solidi B 188, 269 (1995).

${ }^{17}$ Q. W. Mo, T. W. Fan, J. Wu, Z. G. Wang, and Y. Q. Bai, Appl. Phys. Lett. 73, 3518 (1998).

${ }^{18}$ R. Carles, N. Siant-Cricq, J. B. Renucci, and R. J. Nicholas, J. Phys. C 13, 899 (1980).

${ }^{19}$ E. Bedel, R. Carles, A. Zwick, J. B. Renucci, and M. A. Renucci, Phys. Rev. B 30, 5923 (1984).

${ }^{20}$ N. W. McLachlan, Theory and Application of Mathieu Functions (Clarendon, Oxford, 1974).

${ }^{21}$ I. S. Gradshteyn and I. M. Ryzhik, Table of Integrals, Series, and Products (Academic, New York, 1980).

${ }^{22}$ From the electronic archive of Ioffe Physico-Technical Institute (http:// www.ioffe.ru/SVA/NSM/).

${ }^{23}$ J. Groenen, A. Mlayah, R. Carles, A. Ponchet, A. LeCorre, and S. Salaun, Appl. Phys. Lett. 69, 943 (1996).

${ }^{24}$ I. Rasnik, M. J. S. P. Brasil, F. Cerdeira, C. A. C. Mendonça, and M. A. Cotta, J. Appl. Phys. 87, 1165 (2000). 